\title{
Stereotactic aspiration combined with gamma knife radiosurgery for the treatment of cystic brainstem metastasis originating from lung adenosquamous carcinoma: A case report
}

\author{
CHAO DU ${ }^{1}$, ZHAOHUI LI $^{1}$, ZHIJIA WANG ${ }^{2}$, LIPING WANG $^{3}$ and YU TIAN ${ }^{1}$ \\ Departments of ${ }^{1}$ Neurosurgery, ${ }^{2}$ Radiology and ${ }^{3}$ Pathology, \\ China-Japan Union Hospital of Jilin University, Changchun, Jilin 130033, P.R. China
}

Received September 22, 2014; Accepted January 22, 2015

DOI: $10.3892 / \mathrm{ol} .2015 .2968$

\begin{abstract}
Brainstem metastases have a poor prognosis and are difficult to manage. The present study describes the first case of histopathologically-confirmed brainstem metastasis originating from lung adenosquamous carcinoma, and discusses the outcomes of treatment by stereotactic aspiration combined with gamma knife radiosurgery (GKRS). A 59-year-old female presented with a cystic mass $\left(15 \times 12 \times 13 \mathrm{~mm}\right.$; volume, $\left.1.3 \mathrm{~cm}^{3}\right)$ located in the pons, two years following surgical treatment for adenosquamous carcinoma of the lung. The patient received initial GKRS for the lesion in the pons with a total dose of 54.0 Gy, however, the volume of the mass subsequently increased to $3.9 \mathrm{~cm}^{3}$ over a period of three months. Computed tomography-guided stereotactic biopsy and aspiration of the intratumoral cyst were performed, yielding $2.0 \mathrm{~cm}^{3}$ of yellow-white fluid. Histology confirmed the diagnosis of adenosquamous carcinoma. Aspiration provided immediate symptomatic relief, and was followed one week later by repeat GKRS with a dose of $12.0 \mathrm{~Gy}$. The patient survived for 12 months following the repeat GKRS; however, later succumbed to the disease after lapsing into a two-week coma. The findings of this case suggest that stereotactic aspiration of cysts may improve the effects of GKRS for the treatment of cystic brainstem metastasis; the decrease in tumor volume allowed a higher radiation dose to be administered with a lower risk of radiation-induced side effects. Therefore, stereotactic aspiration combined with GKRS may be an effective
\end{abstract}

Correspondence to: Dr Yu Tian, Department of Neurosurgery, China-Japan Union Hospital of Jilin University, 126 Xiantai Street, Changchun, Jilin 130033, P.R. China

E-mail: tianyu@jlu.edu.cn

Abbreviations: SRS, stereotactic radiosurgery; GKRS, gamma knife radiosurgery; MRI, magnetic resonance imaging; $\mathrm{CT}$, computed tomography; MRS, magnetic resonance spectroscopy

Key words: adenosquamous carcinoma, gamma knife radiosurgery, brainstem metastasis, stereotactic aspiration treatment for brainstem metastasis originating from adenosquamous carcinoma.

\section{Introduction}

Brain metastases are common intracranial malignancies, occurring in $15-40 \%$ of adult cancer patients. Only $3-5 \%$ of all brain metastases are located in the brainstem. The primary tumor with the highest rate of brainstem involvement is lung cancer, followed by breast cancer, ovarian cancer, colorectal cancer, renal cell carcinoma and melanoma. Brainstem metastases have a poor prognosis and are particularly challenging to manage; clinical reviews of patients treated for brainstem metastases have reported median survival times ranging from 1-11 months (1-12). Surgical resection is rarely a viable option due to the high risk of developing new neurological deficits or worsening of existing deficits as the majority of drugs which penetrate the blood brain barrier have been found to exhibit low efficacy in this context (5-7). Chemotherapy is also of limited use in brainstem metastases. Stereotactic radiosurgery (SRS) procedures, including gamma knife radiosurgery (GKRS) and linear accelerator (LINAC), have proven to be effective treatment modalities for brainstem metastases (2-21). To the best of our knowledge, the current study reports the first patient with histopathologically-confirmed brainstem metastasis originating from lung adenosquamous carcinoma, and discusses the outcome of stereotactic aspiration and GKRS. Written informed consent was obtained from the patient's family.

\section{Case report}

A 59-year-old Chinese female presented to the Department of Neurosurgery, The Affiliated Hospital of Beihua University (Jilin, China) with a two-week history of vertigo and gait instability on May 19, 2012. Magnetic resonance imaging (MRI) revealed a cystic mass located in the pons. The cystic brainstem tumor initially measured 15x12x13 mm (volume, $1.3 \mathrm{~cm}^{3}$ ), and the cyst wall exhibited annular enhancement (Fig. 1). The patient had undergone surgical treatment for primary lung cancer, pathologically diagnosed as adenosquamous carcinoma, at Jilin Province Cancer Hospital (Changchun, 

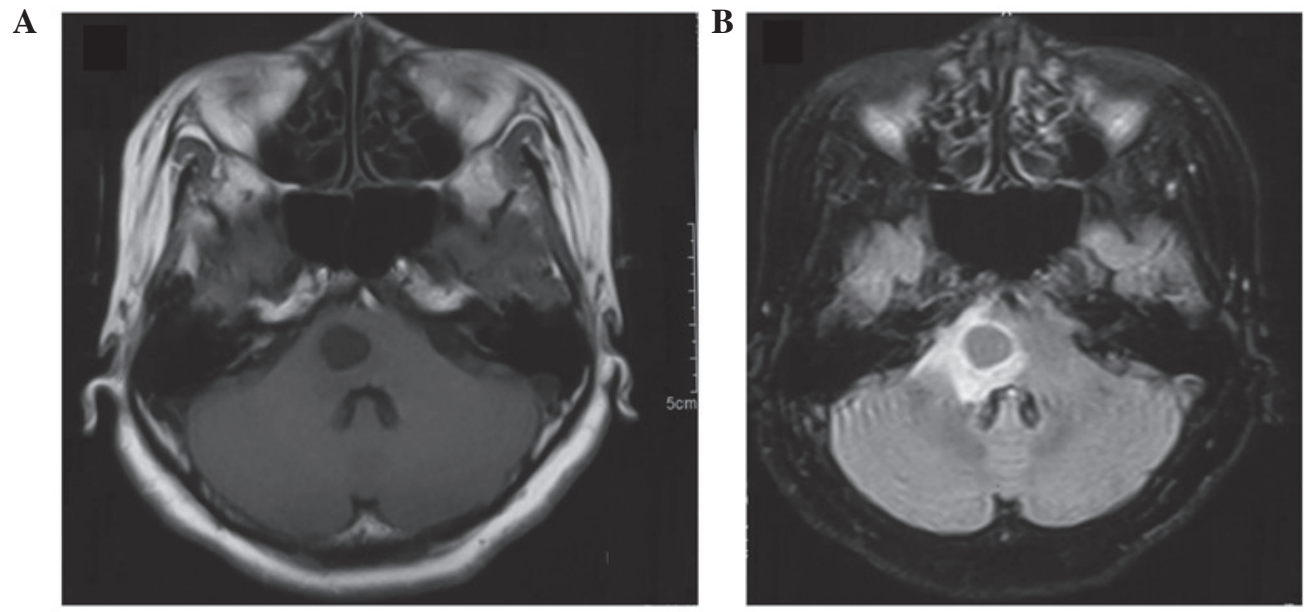

C
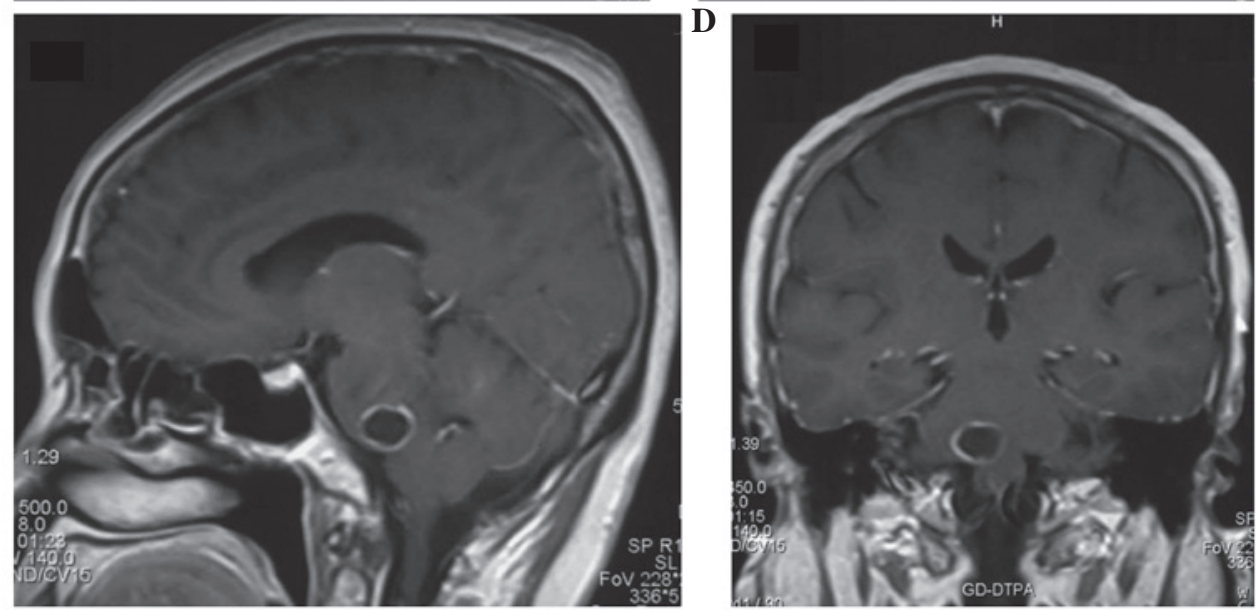

Figure 1. Magnetic resonance images of the cystic brainstem metastasis taken one week after the onset of symptoms. The tumor was $15 \times 12 \times 13 \mathrm{~mm}$ in size (volume, $1.3 \mathrm{~cm}^{3}$ ) prior to the initial gamma knife radiosurgery. (A) Axial T1-weighted image; (B) axial T2-weighted, fluid-attenuated inversion recovery image; (C) sagittal contrast-enhanced T1-weighted image; and (D) coronal contrast-enhanced T1-weighted image. FoV, field of view; GD-TPA, gadolinium diethylenetriamine pentaacetic acid.

China) two years prior to the current presentation. Brainstem metastases were diagnosed based on the clinical and neuroimaging findings, and the patient received initial GKRS for the lesion in the pons over 40 days. The total radiosurgical dose applied to the tumor was $54.0 \mathrm{~Gy}$ in $2 \mathrm{~Gy}$ daily fractions. MRI, three months following the completion of GKRS, revealed that the cystic mass had increased in size to $22 \times 17 \times 19 \mathrm{~mm}$ (volume, $3.9 \mathrm{~cm}^{3}$ ) (Fig. 2). The patient was subsequently referred to the Department of Neurosurgery, China-Japan Union Hospital of Jilin University (Changchun, China) for further treatment.

Upon admission, magnetic resonance spectroscopy (MRS) with peritumoral measurements revealed that the choline/N-acetylaspartate (Cho/NAA) ratio was 3.09 (Fig. 3). Computed tomography-guided stereotactic biopsy and aspiration of the intratumoral cyst, performed three months following the initial GKRS, yielded $2.0 \mathrm{~cm}^{3}$ of yellow-white fluid (Fig. 4), and a smear of the hydatid fluid showed atypical cells (Fig. 5). Histopathology revealed the biopsy specimen to be a metastatic adenosquamous carcinoma originating from the lung. The sample exhibited similar hematoxylin and eosin morphology to lung adenosquamous carcinoma with predominant well-differentiated adenocarcinoma associated with heterologous elements of squamous cell carcinoma. An
MRI scan for gamma knife radiosurgery planning conducted following stereotactic aspiration showed that the cystic tumor in the pons had decreased in size to $18 \times 15 \times 14 \mathrm{~mm}$ (volume, $1.9 \mathrm{~cm}^{3}$; Figure 6). The patient's vertigo and gait instability improved within three days following the aspiration, and a second GKRS was performed one week later (dose, $12.0 \mathrm{~Gy}$ ). Following discharge from hospital, the patient was alert and the neurological symptoms had resolved. A further MRI scan was performed five months subsequent to the repeat GKRS, revealing that the brainstem metastasis had reduced to $12 \times 13 \times 14 \mathrm{~mm}$ (volume, $1.2 \mathrm{~cm}^{3}$ ), without severe radiation-induced edema (Fig. 7). MRI also indicated that the brainstem metastasis was well controlled. The patient survived for 12 months following the repeat GKRS, however, later succumbed to the disease after lapsing into a two-week coma following the development and progression of new brain metastases.

\section{Discussion}

A number of reports have described the treatment of brainstem metastasis, including pontine metastasis (1-11). The most common site of primary malignancy in patients with metastases to the brainstem is the lungs; the majority of such malignancies 
A
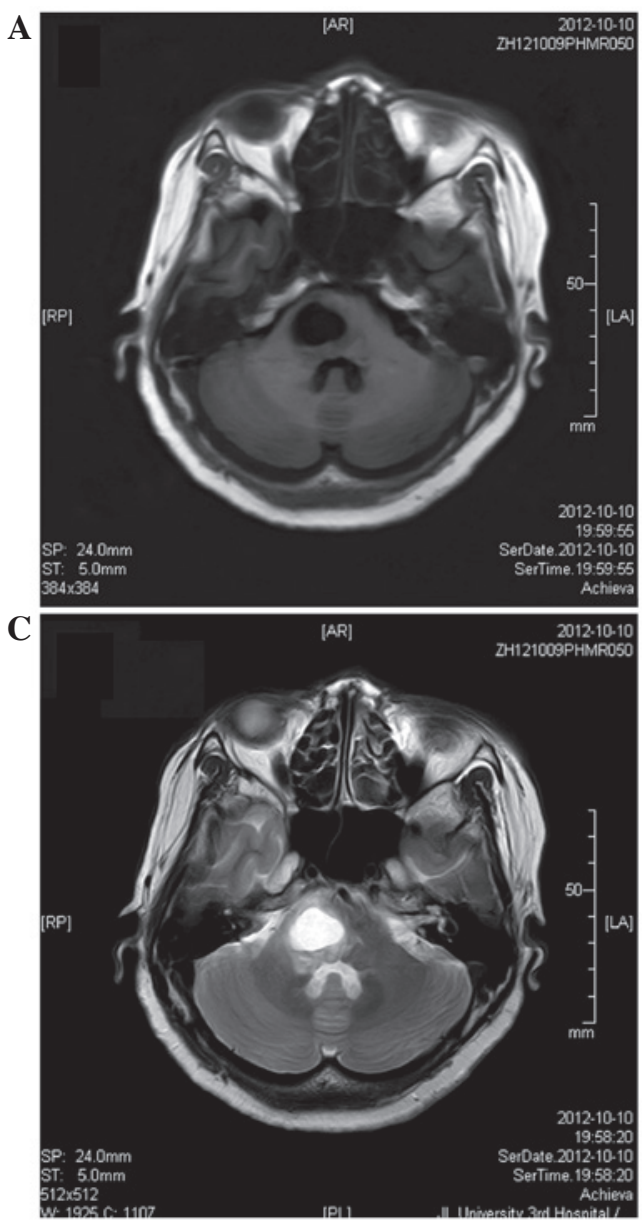

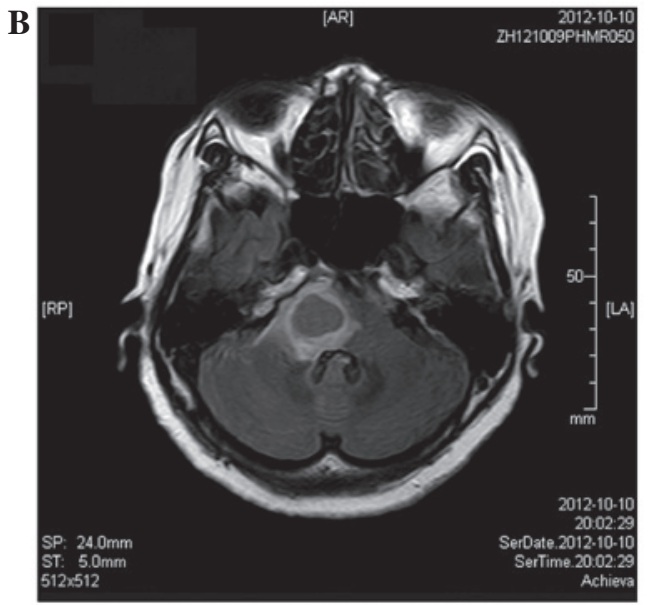

D

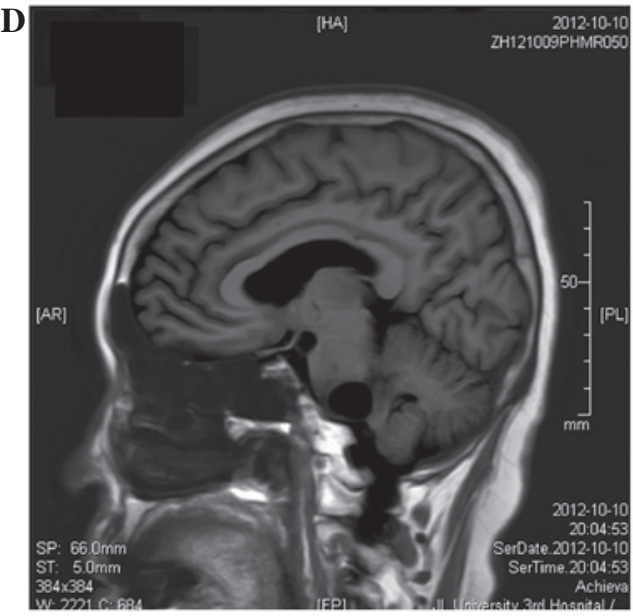

Figure 2. Magnetic resonance images obtained three months following the initial gamma knife radiosurgery. The cystic mass increased in size to $22 x 17 x 19$ mm (volume: $3.9 \mathrm{~cm}^{3}$ ). (A) Axial T1-weighted image; (B) axial T2-weighted, fluid-attenuated inversion recovery image; (C) axial diffusion-weighted image; and (D) sagittal T1-weighted image.

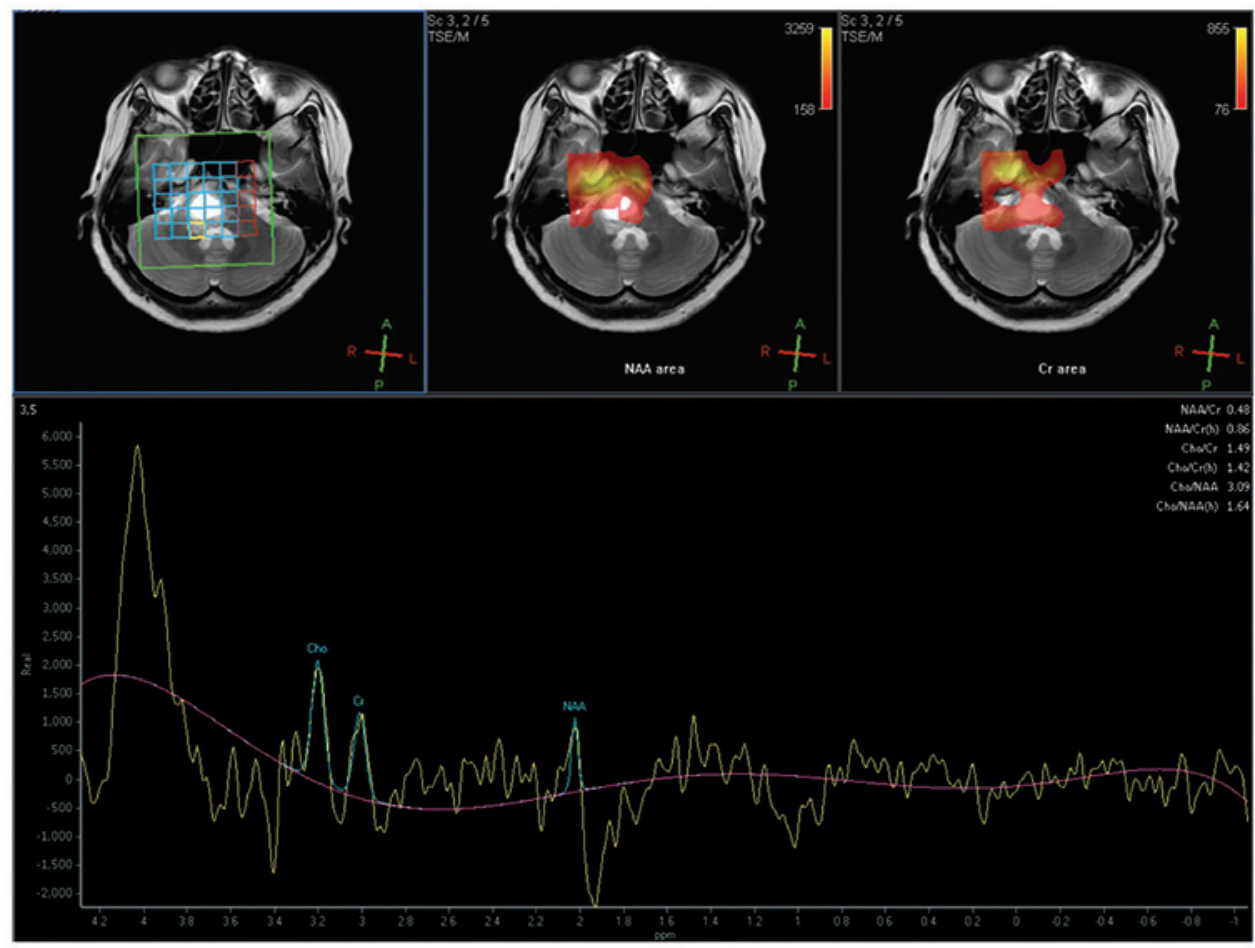

Figure 3. Images from magnetic resonance spectroscopy obtained three months following the initial gamma knife radiosurgery. The yellow shading indicates peritumoral edema. The choline/N-acetylaspartate ratio was 3.09. A, anterior; Cho, choline; Cr, creatine; L, left; NAA, N-acetylaspartate; P, posterior; PPM, parts per million; R, right. 

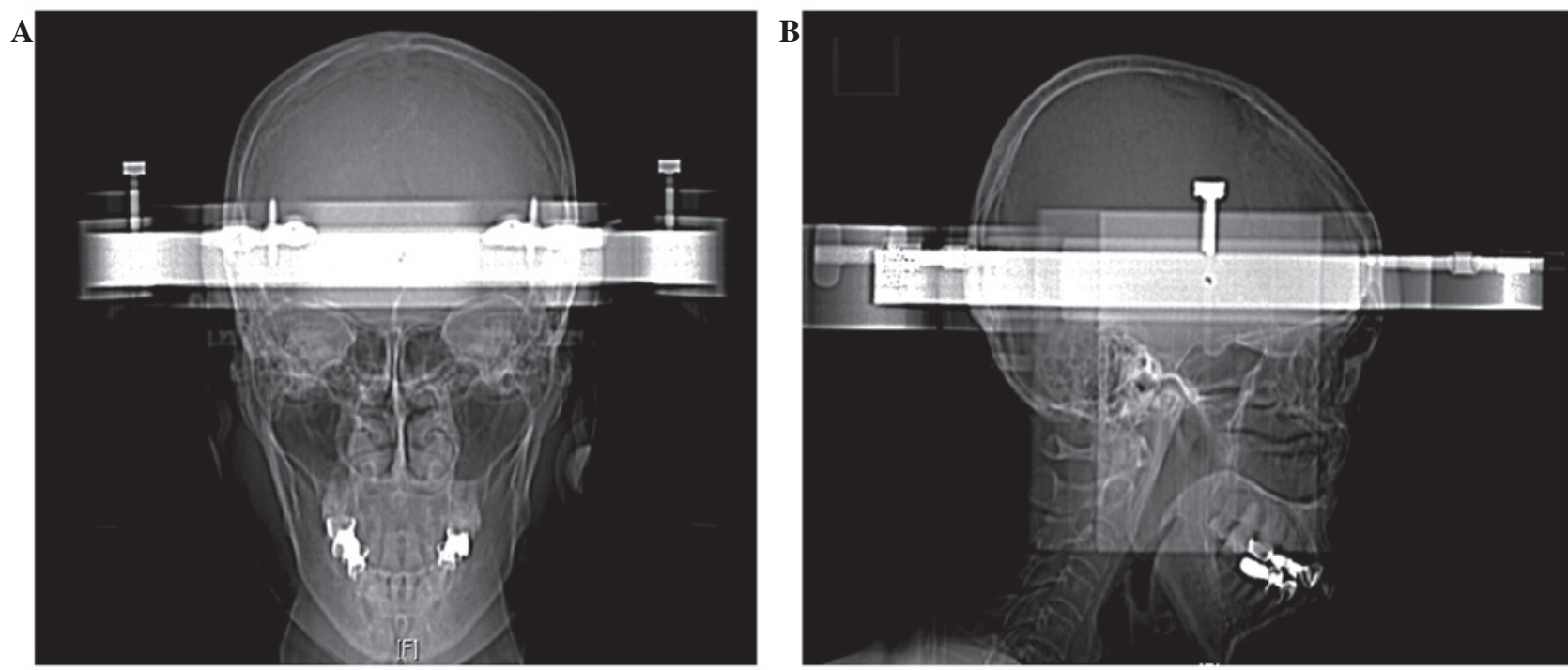

Figure 4. Computed tomographic images showing the setup for stereotactic aspiration. (A) Antero-posterior view; (B) lateral view.
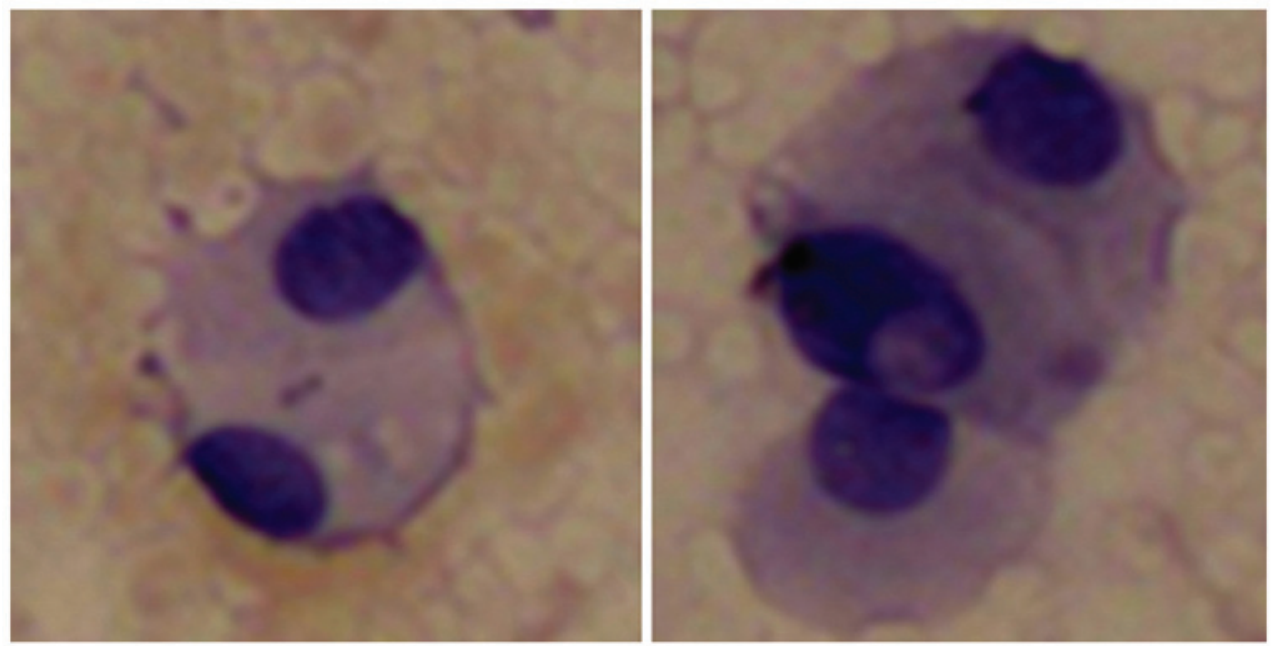

Figure 5. Smear of the cystic fluid showing atypical cells.
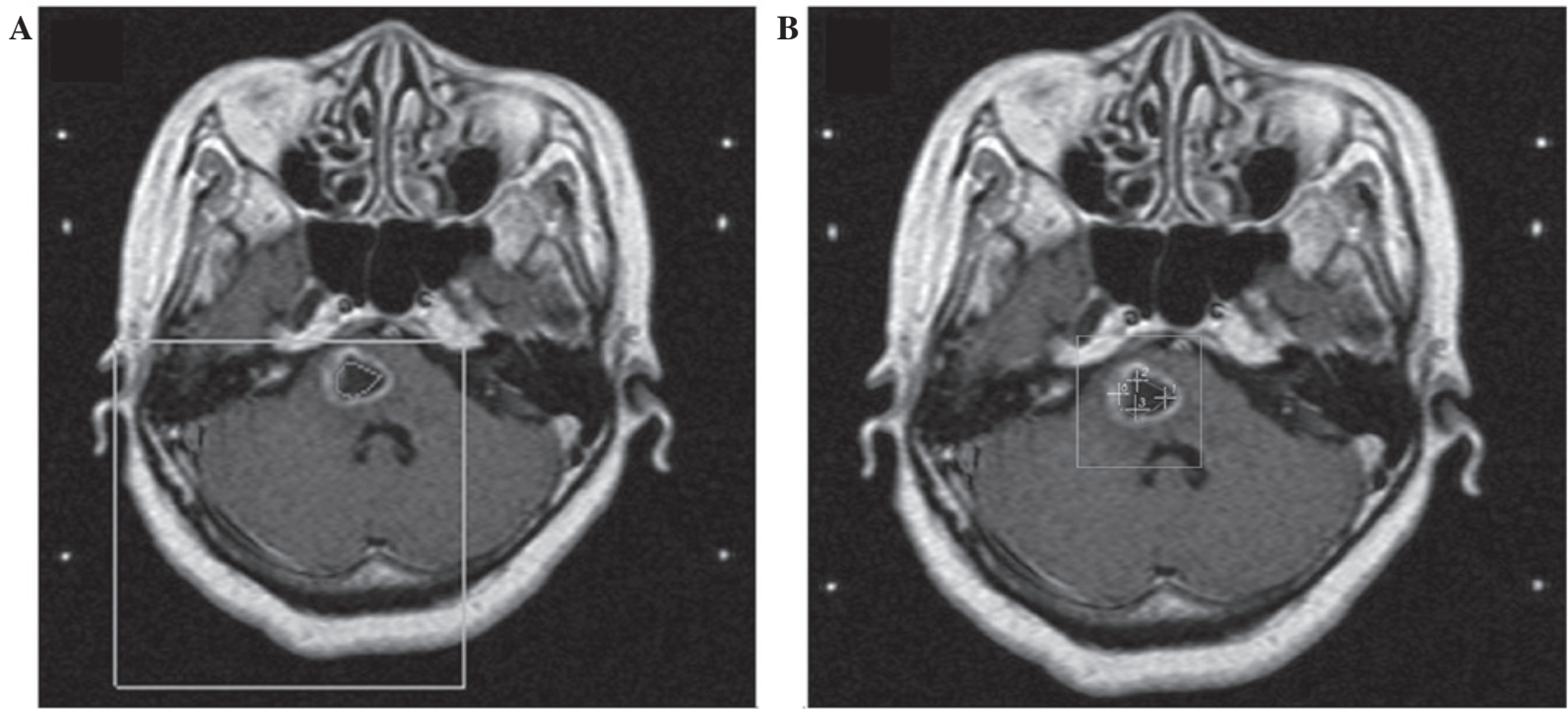

Figure 6. Magnetic resonance images obtained one week following stereotactic aspiration surgery. The tumor decreased in size to $18 \times 15 \times 14 \mathrm{~mm}\left(\right.$ volume, $\left.1.9 \mathrm{~cm}^{3}\right)$, (A) Axial T1-weighted images. (B) Axial T1-weighted images showing dose planning of the tumor for gamma knife radiosurgery. 
A

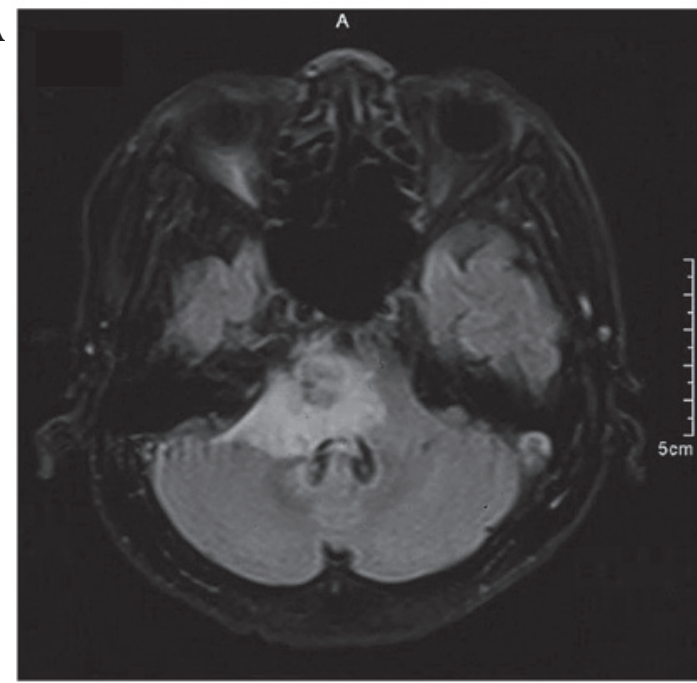

B

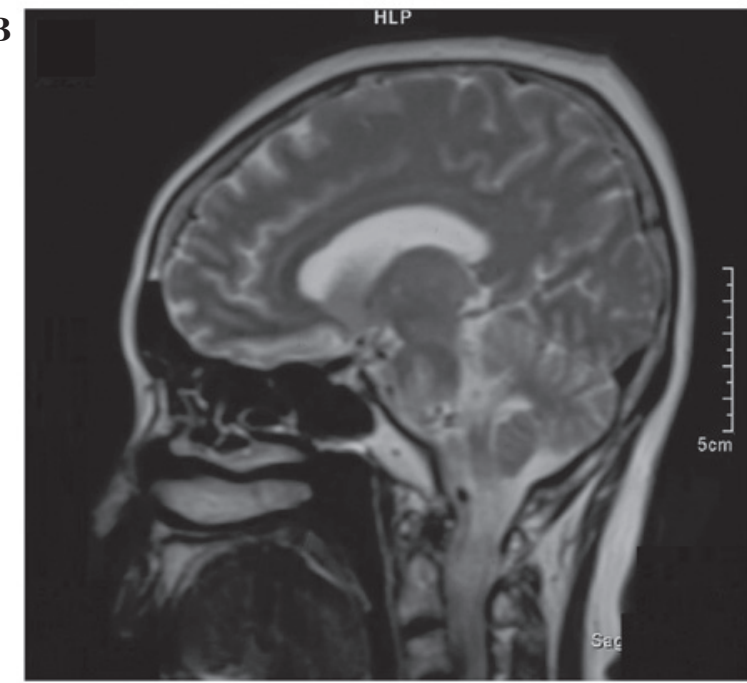

Figure 7. Magnetic resonance images obtained five months following stereotactic aspiration. The tumor size was $12 \mathrm{x} 13 \mathrm{x} 14 \mathrm{~mm}\left(\mathrm{volume}, 1.2 \mathrm{~cm}{ }^{3}\right.$ ). New metastases are visible in the cerebellum. (A) Axial T1-weighted, fluid-attenuated inversion recovery image and (B) sagittal T2-weighted image.

are adenocarcinomas $(6,7,9,10)$ and, less commonly, squamous cell carcinomas (8). SRS is the preferred treatment option for brainstem metastases due to its provision of adequate local control with low morbidity. Accurate targeting of the tumor may limit damage to the surrounding healthy brain tissue, thereby mitigating the neurological decline $(3,6,15)$. Between 1999 and 2014, >20 studies reported the clinical characteristics, radiation doses and outcomes of brainstem metastases following SRS treatment (Table I). In these reports, the median survival time of patients with brainstem metastases ranged from 4-16.8 months (2-21).

In the present case, the patient was initially diagnosed based on clinical and neuroimaging assessments performed at the Department of Neurosurgery, The Affiliated Hospital of Beihua University. Consistent with the standard treatment of irradiation of brain metastases, the patient underwent GKRS of the brainstem metastasis over 40 days with a total dose of 54.0 Gy. This dose was deemed appropriate based on the study by Maranzano et al (22), who reported that the brain tolerated a single course of radiotherapy of 50-60 Gy in 2 Gy daily fractions. However, in the current case, MRI performed three months following GKRS showed an increase in the size of the cystic mass. The patient was therefore referred to the China-Japan Union Hospital of Jilin University for further treatment.

As part of the clinical evaluation, it was critical to determine whether the mass was a metastatic growth or a high-grade glioma. Server et al (23) reported that MRS may be utilized to differentiate high-grade glioma from metastases, particularly when the performed in conjunction with measurement of the peritumoral $\mathrm{Cho/NAA}$ ratio, which has a high sensitivity (100\%). In the current case, the results of these tests indicated the presence of a metastatic carcinoma.

Stereotactic aspiration is a minimally invasive technique. It is widely used to treat brain abscesses (24), and is an important component of the multimodal treatment of cystic craniopharyngiomas (25). Several studies have also applied stereotactic aspiration for the treatment of glial and metastatic brain tumors (26-28). Higuchi et al (26) used a procedure in which stereotactic aspiration followed by GKRS on the same day provided good tumor control in 25 patients with cystic metastases. Park et al (27) performed stereotactic aspiration, which was followed by GKRS, in 24 patients with cystic metastatic brain tumors. Following treatment, 13 patients $(54.2 \%)$ had good tumor control, five patients $(20.8 \%)$ exhibited local tumor progression, and six patients $(25.0 \%)$ had remote progression. The overall median survival time was 17.8 months, and no cases of brainstem metastasis were identified. To the best of our knowledge, Aung et al (29) were the first to report a patient with adenocarcinoma of the right main bronchus that disseminated to the pons, left cerebral peduncle, and liver. In this case, CT-guided stereotactic aspiration of the cystic pontine lesion was performed and a catheter inserted in the cyst cavity was connected to a subgaleal Ommaya reservoir for further aspiration and decompression. Although these procedures alleviated the patient's clinical symptoms, the patient succumbed to the disease three weeks following the stereotactic surgery, after becoming comatose, with jaundice and ascites. Matsumoto et al (30) reported a patient with a cystic metastasis in the midbrain that was successfully treated by brachytherapy following stereotactic biopsy and aspiration of the intratumoral cyst. The authors reported that stereotactic aspiration of cystic lesions provides clinical improvement, and brachytherapy prevents cyst recurrence. In our previous report (31), we treated a brainstem cystic glioma by combining stereotactic aspiration with GKRS, with a favorable outcome. Therefore, we speculated that stereotactic aspiration surgery may improve the effect of GKRS treatment of cystic brainstem glioma.

In the present case, however, the cystic mass progressed and disease control was lost following the initial GKRS. As the results from prior reports and our previous case indicated that stereotactic aspiration of the cystic mass may be an effective treatment, the patient underwent CT-guided stereotactic biopsy, which yielded $2.0 \mathrm{~cm}^{3}$ of cystic fluid. Histopathological analysis of the exudate confirmed the origin as primary adenosquamous carcinoma of the lung. Following stereotactic aspiration, GKRS was repeated, reducing the volume of the mass to $1.9 \mathrm{~cm}^{3}$. An 


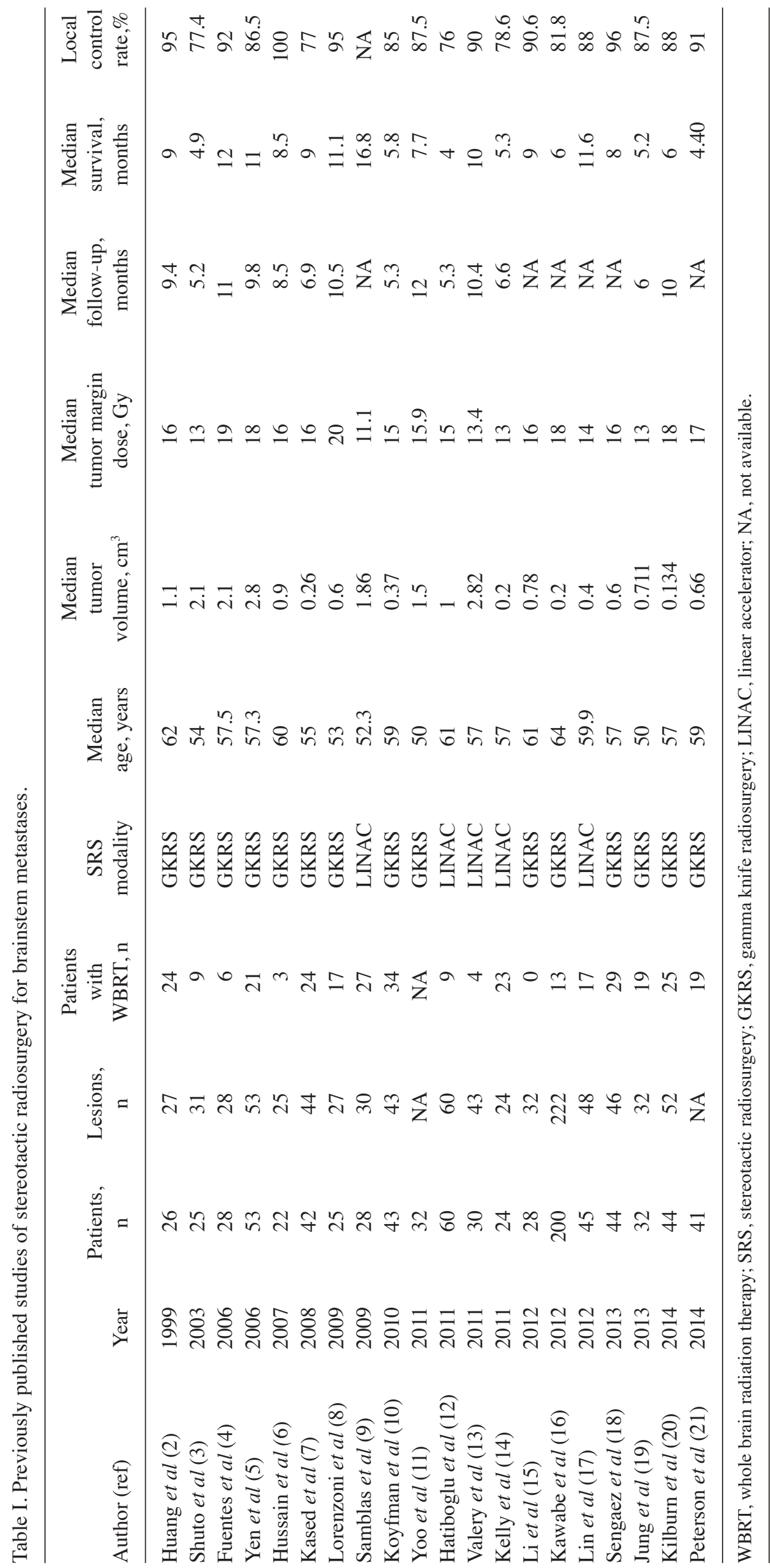


MRI scan performed five months following the repeat GKRS revealed a controlled mass of $1.2 \mathrm{~cm}^{3}$ without substantial radiotherapy-induced edema. However, a second metastatic lesion was detected in the cerebellum of this patient following the second GKRS. The patient survived for 17 months following the first MRI (12 months after the repeat GKRS).

Adenosquamous carcinoma is an uncommon lung cancer, accounting for $0.4-4 \%$ of all primary lung cancers, in which $\geq 10 \%$ of the tumor volume contains adenocarcinoma and squamous cell carcinoma components. Adenosquamous carcinomas have a significantly poorer prognosis compared with adenocarcinomas and squamous cell carcinomas. Brain metastasis is common in patients with adenosquamous carcinoma of the lung, however, metastasis to the brainstem from this carcinoma is extremely uncommon (32-35), and the present case is the first in which adenosquamous carcinoma of the lung with metastasis to the brainstem has been reported. Furthermore, the current case is the first to report stereotactic aspiration and GKRS for the treatment of a cystic brainstem metastasis. This treatment provided good local tumor control and the patient survived for 12 months following the procedure. The patient's survival time of 17 months from initial diagnosis is longer than that of patients reported elsewhere. This indicates that the use of multiple treatment modalities, including stereotactic aspiration combined with GKRS, may extend the survival time of patients with cystic brainstem metastases.

In conclusion, this case suggests that cyst drainage by stereotactic aspiration improves the effects of GKRS in the treatment of cystic brainstem metastasis, by reducing the tumor burden and enhancing local tumor control. To date, there are no evident-based guidelines for the treatment of brainstem metastasis; for the brainstem metastasis with cystic components, multimodality strategy combing stereotactic aspiration with GKRS may be considered. Furthermore, large series of patients with cystic brainstem metastasis should be studied to make the final disgnosis.

\section{References}

1. Lamm AF, Elaimy AL, Lamoreaux WT, et al: A review of the clinical outcomes for patients diagnosed with brainstem metastasis and treated with stereotactic radiosurgery. ISRN Surg 2013: 652895, 2013.

2. Huang CF, Kondziolka D, Flickinger JC and Lunsford LD: Stereotactic radiosurgery for brain stem metastases. J Neurosurg 91: 563-568, 1999.

3. Shuto T, Fujino H, Asada H, et al: Gamma knife radiosurgery for metastatic tumours in the brain stem. Acta Neurochir (Wien) 145 755-760, 2003.

4. Fuentes S, Delsanti C, Metellus P, et al: Brain stem metastases: management using gamma knife radiosurgery. Neurosurgery 58 : 37-42, 2006.

5. Yen CP, Sheehan J, Patterson G and Steiner L: Gamma knife surgery for metastatic brain stem tumors. J Neurosurg 105: 213-219, 2006.

6. Hussain A, Brown PD, Stafford SL and Pollock BE: Stereotactic radiosurgery for brain stem metastases: Survival, tumor control, and patient outcomes. Int J Radiat Oncol Bio Phys 67: 521-524, 2007.

7. Kased N, Huang K, Nakamura JL, et al: Gamma knife radiosurgery for brainstem metastases: the UCSF experience. J Neurooncol 86: 195-205, 2008.

8. Lorenzoni JG, Devriendt D, Massager N, et al: Brain stem metastases treated with radiosurgery: prognostic factors of survival and life expectancy estimation. Surg Neurol 71: 188-196, 2009.

9. Samblás JM, Sallabanda K, Bustos JC, et al: Radiosurgery and whole brain therapy in the treatment of brainstem metastases. Clinical Transl Oncol 11: 677-680, 2009.
10. Koyfman SA, Tendulkar RD, Chao ST, et al: Stereotactic radiosurgery for single brainstem metastases: the cleveland clinic experience. Int J Radiat Oncol Bio Phys 78: 409-414, 2010.

11. Yoo TW,Park ES, Kwon do H and Kim CJ: Gammaknife radiosurgery for brainstem metastases. J Korean Neurosurg Soc 50: 299-303, 2011.

12. Hatiboglu MA, Chang EL, Suki D, et al: Outcomes and prognostic factors for patients with brainstem metastases undergoing stereotactic radiosurgery. Neurosurgery 69: 796-806, 2011.

13. Valery CA, Boskos C, Boisserie G, et al: Minimized doses for linear accelerator radiosurgery of brainstem metastasis. Int J Radiat Oncol Biol Phys 80: 362-368, 2011.

14. Kelly PJ, Lin YB, Yu AY, et al: Linear accelerator-based stereotactic radiosurgery for brainstem metastases: the Dana-Farber/Brigham and Women's Cancer Center experience. J Neurooncol 104: 553-557, 2011.

15. Li Y, Xu D, Zhang Z, et al: Gamma knife surgery for brainstem metastases. J Neurosurg 117: 13-16, 2012.

16. Kawabe T, Yamamoto M, Sato Y, et al: Gamma Knife surgery for patients with brainstem metastases. J Neurosurg 117: 23-30, 2012.

17. Lin CS, Selch MT, Lee SP, et al: Accelerator-based stereotactic radiosurgery for brainstem metastases. Neurosurgery 70: 953-958, 2012.

18. Sengöz M, Kabalay IA, Tezcanlı E, Peker S and Pamir N: Treatment of brainstem metastases with gamma-knife radiosurgery. J Neurooncol 113: 33-38, 2013.

19. Jung EW, Rakowski JT, Delly F, et al: Gamma Knife radiosurgery in the management of brainstem metastases. Clin Neurol Neurosurg 115: 2023-2028, 2013.

20. Kilburn JM, Ellis TL, Lovato JF, et al: Local control and toxicity outcomes in brainstem metastases treated with single fraction radiosurgery: is there a volume threshold for toxicity? J Neurooncol 117: 167-174, 2014.

21. Peterson HE, Larson EW, Fairbanks RK, et al: Gamma knife treatment of brainstem metastases. Int J Mol Sci 15: 9748-9761, 2014.

22. Maranzano E, Trippa F, Pacchiarini D, et al: Re-irradiation of brain metastases and metastatic spinal cord compression: clinical practice suggestions. Tumori 91: 325-330, 2005.

23. Server A, Josefsen R, Kulle B, et al: Proton magnetic resonance spectroscopy in the distinction of high-grade cerebral gliomas from single metastatic brain tumors. Acta Radiol 51: 316-325, 2010.

24. Lü Y, Li C, Liu M, et al: MRI-guided stereotactic aspiration of brain abscesses by use of an optical tracking navigation system. Acta Radiol 55: 121-128, 2014.

25. Hofmann BM, Höllig A, Strauss C, Buslei R, Buchfelder M and Fahlbusch R: Results after treatment of craniopharyngiomas: further experiences with 73 patients since 1997. J Neurosurg 116: 373-384, 2012.

26. Higuchi F, Kawamoto S, Abe Y,Kim P and Ueki K: Effectiveness of a 1-day aspiration plus Gamma Knife surgery procedure for metastatic brain tumor with a cystic component. J Neurosurg 117: 17-22, 2012.

27. Kim IY, Yun SJ, Lee JS, et al: Brain metastasis from extramammary Paget's disease of the scrotum. J Clin Neurosci 21: 694-696, 2014.

28. Park WH, Jang IS, Kim CJ and Kwon do H: Gamma knife radiosurgery after stereotactic aspiration for large cystic brain metastases. J Korean Neurosurg Soc 46: 360-364, 2009.

29. Aung TH, Kwok CK, Mark YF and Mark EG: Computed tomography-guided stereotactic aspiration and biopsy of a cystic metastatic adenocarcinoma of the pons. Hong Kong Med J 3: 232-235, 1997.

30. Matsumoto K, Tada E, Tamesa N, Tomita S and Ohmoto T: Stereotactic brachytherapy for a cystic metastatic brain tumor in the midbrain. Case report. J Neurosurg 88: 141-144, 1998.

31. Zhao X, Du C and Tian Y: Results Of Repeat Gamma Knife Treatment: Stereotactic Aspiration Surgery Can Improve the Effect of Gamma Knife Treatment for Cystic Small Brainstem Glioma: Case Report. Neurosurgery Quarterly 22: 22-25, 2012.

32. Nakagawa K, Yasumitu T, Fukuhara K, Shiono H and Kikui M: Poor prognosis after lung resection for patients with adenosquamous carcinoma of the lung. Ann Thorac Surg 75: 1740-1744, 2003.

33. Gawrychowski J,Bruliński K, MalinowskiEand Papla B: Prognosis and survival after radical resection of primary adenosquamous lung carcinoma. Eur J Cardiothorac Surg 27: 686-692, 2005.

34. Cooke DT, Nguyen DV, Yang Y, et al: Survival comparison of adenosquamous, squamous cell and adenocarcinoma of the lung after lobectomy. Ann Thorac Surg 90: 943-948, 2010.

35. Filosso PL, Ruffini E, Asioli S, et al: Adenosquamous lung carcinomas: a histologic subtype with poor prognosis. Lung Cancer 74: 25-29, 2011. 